\title{
RETRACTED ARTICLE: Introduction
}

\section{Ryan Coder $^{1} \cdot$ Kyle DeMars $^{2} \cdot$ Marcus Holzinger $^{3} \cdot$ Lauchie Scott $^{4}$}

Published online: 13 July 2020

(C) American Astronautical Society 2020

The Editor-in-Chief has retracted this Editorial which was published prematurely. All authors agree with this retraction.

Supplementary Information The online version contains supplementary material available at https://doi. org/10.1007/s40295-020-00225-0.

\author{
Lauchie Scott \\ Lauchie.scott@drdcrddc.gc.ca \\ Ryan Coder \\ ryan.coder.1@us.af.mil \\ Kyle DeMars \\ demars@tamu.edu \\ Marcus Holzinger \\ Marcus.holzinger@colorado.edu
}

1 Air Force Research Laboratory, Ohio, USA

2 Texas A\&M University, College Station, TX 77843-3141, USA

3 University of Colorado - Boulder, 429 UCB, Boulder, Colorado 80309-0429, USA

4 Defence Research and Development Canada, 3701 Carling Ave, Ottawa, ON K1A 0Z4, Canada 